\title{
Activation of JNK and p38 in rat hippocampus after kainic acid induced seizure
}

\author{
Song Hee Jeon', Yong Sik Kim², \\ Chang-Dae Bae ${ }^{1}$ and Joo-Bae Park ${ }^{1,3}$
}

${ }^{1}$ Department of Molecular Cell Biology and Samsung Biomedical
Research Institute, Sunkyunkwan University School of Medicine,
Suwon 440-746, Korea
${ }^{2}$ Department of Psychiatry, Seoul National University College of
Medicine, Seoul 110-799, Korea
${ }^{3}$ Corresponding author: Tel, +82-31-299-6130;
Fax, +82-31-299-6149; E-mail, jbpark@med.skku.ac.kr

Accepted 11 December 2000

Abbreviations: KA, kainic acid; JNK, c-Jun N-terminal kinase; MAPK, mitogen-activated protein kinase; ERK, extracellular signalregulated kinase; MKK, MAPK kinase; ECL, enhanced chemiluminescence

\begin{abstract}
Kainic acid, an analogue of glutamate, causes limbic seizures and induces cell death in the rat brain. We examined the activation of MAPK family kinases; ERKs, JNKs and p38 kinase in rat hippocampus after KA treatment. Activation of all three kinases were observed at $\mathbf{3 0} \mathrm{min}$ after the treatment, but, in contrary to ERK phosphorylation, which lasted up to $3 \mathrm{~h}$, the phosphorylation of JNK and p38 returned to the basal level by $2 \mathrm{~h}$. The phosphorylation of upstream kinases for the MAPK family was distinct. The phosphorylation of MEK1 clearly increased at 30 min but diminished rapidly thereafter. The phosphorylation of MKK6 was also increased but reached peak at $\mathbf{2} \mathrm{h}$ after KA treatment. However, the phosphorylation of other upstream kinases, SEK1 and MKK3, gradually decreased to $3 \mathrm{~h}$ after KA treatment. These results indicate that the KA activates all of the three MAPK family kinases with different time patterns and suggest the possibility that MKK3 and MKK6, and SEK1 may not be the upstream kinases for p38 and JNK in rat hippocampus.
\end{abstract}

Keywords: hippocampus, kainic acid, ERK, JNK, p38, phosphorylation

\section{Introduction}

Glutamate has been known to play crucial roles in syn- aptic plasticity (Nicoll et al., 1988) and excitotoxic cell death (Choi, 1988). Glutamate receptors are classified as NMDA, AMPA and KA receptors based on their prototypical agonists. We have been studying the induction of immediate early genes such as c-fos and c-jun and also activation of MAPK family kinases after electrically-induced seizures. Various glutamates are known to activate MAPKs in cell lines. However, the role of these glutamate receptors in the activation of MAPKs in rat brain after electrically-induced seizures are not clearly understood. The 42 and 44 kD MAPKs are activated in rat hippocampus after kainic acid (KA) treatment (Kim et al., 1994). KA, an analogue of glutamate, induces limbic seizures in rat, and KA-induced seizure has been used as a model for studying human seizures. $K A$ is known to induce the expression of various immediate early genes such as c-fos and c-jun and activates signaling molecules in rat brain. However, the KA-induced signal transduction pathway leading to the induction of immediate early gene expression and excitotoxic cell death are largely unknown in vivo.

The c-Jun N-terminal kinases (JNKs), also called stressactivated protein kinase (SAPK), is one of the MAPK superfamily which is involved in the stress response and cell death. Another member of MAPK superfamily, p38 is responsive to osmotic shock and cell death as well as many stressful stimuli (Widmann et al., 1999). In neuronal cell lines, the MAPKs are activated by depolarization, growth factors, glutamates and phorbol ester and involved in the cellular differentiation or proliferation, and promotes survival of cells (Seger and Krebs, 1995). Although, an each member of MAPK family has a separate signaling pathway, there are evidences that they converge on some points of cascade to balance the separate pathways (Cheng and Feldman, 1998).

The members of MAPK family are activated by dual specific kinases known as MAPK kinases (MKKs), which in turn is activated by MAPK kinase kinases (MKKKs). To date, about 10 different MKKs have been cloned in mammals. Such diversity of MKKs is believed to enable cells to properly respond to various stimuli. MEK1 and MEK2 are known to activate ERKs, SEK1 (MKK4/JNKK1) and JNK2. MKK7 is known to activate JNK. SEK1, MKK3 and MKK6 are known to activate p38 (Widmann et al., 1999).

Although, the consequences of the activation of MAPK family are not clear as yet, it has been generally accepted that in neuronal cells, JNK and p38 are important in cell death, whereas ERKs are involved in cell differentiation or survival (Xia et al., 1995). However, the functional independence of each members of MAPK family 
is not absolute and cross talks between them may occur (Robinson and Cobb, 1997). Previously, we reported the activation of 42 and 44 kD MAPK (ERK1 and ERK2) in accompanying seizure activities after KA treatment in rat hippocampus (Kim et al., 1994). In the present study, we examined the activation of two other members of MAPK family, JNK and p38 and their upstream MAPK kinases by $\mathrm{KA}$ in rat hippocampus.

\section{Materials and Methods}

Male Sprague-Dawley rats ranging 150-200 gm in weight were used. Animals were treated in accordance with the $\mathrm{NIH}$ guide for the care and use of Laboratory Animals. After injection of KA (12 mg/kg, i.p.; Sigma), the animals were decapitated at the given time points $(0.5,1,2,3 \mathrm{~h}$ after KA treatment). The hippocampi were dissected out on ice and homogenized immediately with glass-teflon homogenizer in 10 volume of the ice-cold homogenization buffer (25 mM Tris pH 7.5, $1 \mathrm{mM}$ EGTA, $2 \mathrm{mM}$ EDTA, $50 \mathrm{mM} \mathrm{NaF}, 1 \mathrm{mM} \mathrm{Na} \mathrm{VO}_{4}, 10 \mathrm{mM}$ sodium pyrophosphate, 0.2\% NP-40, $0.1 \mathrm{mM}$ PMSF, $1 \mathrm{mM}$ DTT, $10 \mu \mathrm{g} / \mathrm{ml}$ aprotinin, $10 \mu \mathrm{g} / \mathrm{ml}$ leupeptin, $10 \mu \mathrm{g} / \mathrm{ml}$ pepstatin), and centrifuged at $10,000 \times g$ for $10 \mathrm{~min}$, at $4^{\circ} \mathrm{C}$. The supernatant was boiled with Laemmli's sampling buffer. Eighty micrograms of the hippocampal lysate were electrophoresed in 8\% SDS-polyacrylamide gel and then transferred to nitrocellulose membranes. The membranes were incubated with anti-phospho-JNK (Thr183/ Tyr185), anti-phospho-SEK1 (Thr223), anti-phosphop38 (Thr180/Tyr182), anti-phospho-MKK3/6 (Ser189/ Ser207), anti-phospho-MEK1/2 (Ser217/221) and antiphospho-p44/42 ERK (Thr202/Tyr204) antibodies (New England Biolabs). After visualization by ECL (Pierce), the membranes were reprobed with antibodies (Santa Cruz) againt JNK, SEK1, p38, MKK6, ERK and MEK.

\section{Results}

Kainic acid treatment increased the phosphorylation of all three MAPK family kinases and induced an activation of 42 and $44 \mathrm{kD}$ ERKs (Kim et al., 1994). We confirmed that KA-induced phosphorylation of ERKs coincide well with the reported activation; reaching peak at $30 \mathrm{~min}$ after treatment and then gradually decreasing (Figure $1 \mathrm{~A}$, left). Even $3 \mathrm{~h}$ after treatment, the phosphorylation did not return to the basal level.

In rat brain, many isoforms of JNK are expressed (Casanova et al., 1996), and most of the isoforms can be grouped into 46 and $54 \mathrm{kD}$ JNKs by molecular weight. Among these two forms, we only observed the increase of the phosphorylation of p46 JNK in rat hippocampus after KA treatment. The phosphorylation of p46 JNK was peaked at 30 min after KA treatment along
A.

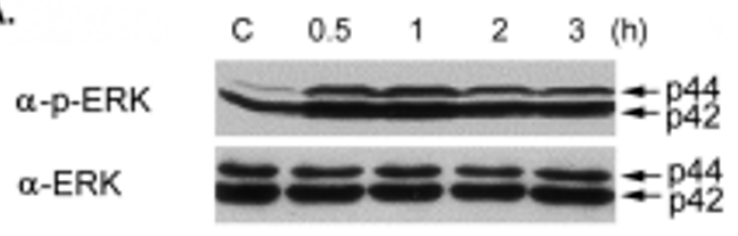

$a-p-M E K 1$

$a-M E K 1$

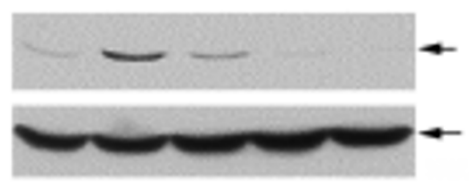

B.

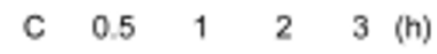

$\alpha$-p-JNK
$\alpha$-JNK

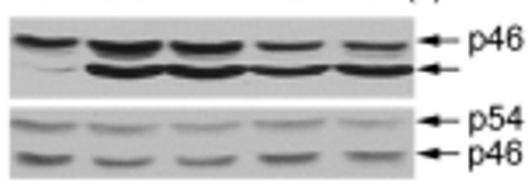

$\alpha$-p-SEK1

$\alpha$-SEK1

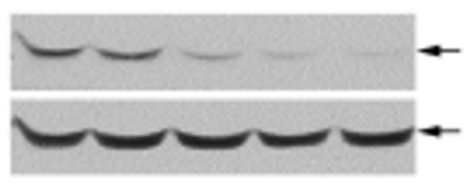

C.

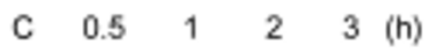

$\alpha-p-p 38$
$\alpha-p 38$

$\alpha-p-M K K 3$

$\alpha-p-M K K 6$

$\alpha-M K K 6$

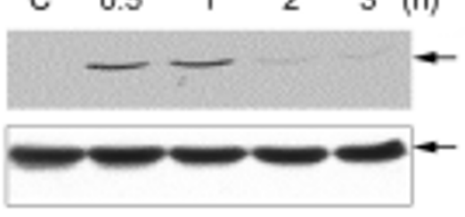

Figure 1. Phosphorylation of ERK, MEK, p38, MKK3/6, JNK and SEK1 after KA administration in rat hippocampus. After KA injection (i.p. $12 \mathrm{mg} / \mathrm{kg}$ ), rats were decapitated at the indicated times. Hippocampus was homogenized and subjected to SDS-PAGE. The proteins were transferred to nitrocellulose membranes and the membranes were immunoblotted with antibodies against phospho-p44/42 MAPK, phospho-MEK1/2 (A), phospho-JNK, phospho-SEK1 (B), phospho-p38 and phospho-MKK3/6 (C). After visualization by ECL, the membranes were reprobed with anti-ERK, -MEK, -JNK, -SEK1, -p38 and -MKK6 antibodies. The arrow in top pannel of Figure B indicates phosphorylated ERK, which cross-reacted with anti-phospho-JNK antibody.

with seizure onset, indicated by wet dog shake, and returned to the basal level by $2 \mathrm{~h}$ (Figure 1B, left). The average increase of JNK phosphorylation at peak level was about $70 \%$. Although p54 JNK was expressed in hippocampus, the phosphorylation of p54 JNK was hardly detectable in rat hippocampus. These result strongly suggested that the JNK isoforms are regulated differently through different upstream signaling pathways (Gupta et al., 1996). The phosphorylation of p38, another 
member of MAPK family, was increased at 30 min after $\mathrm{KA}$ treatment in rat hippocampus. The phosphorylation lasted up to $1 \mathrm{~h}$ and returned to the basal level by $2 \mathrm{~h}$ (Figure 1C, left). The temporal pattern of p38 phosphorylation was same as JNK

Examination of the activation of upstream kinases of MAPK family kinases showed that upstream kinase MEK was activated after KA treatment in rat hippocampus along with the phosphorylation of ERKs. The phosphorylation of MEK was increased to maximum level at 30 min after KA treatment, but it did not prolong; returned to the basal level by $1 \mathrm{~h}$ (Figure 1A, right). SEK1 is known as the upstream kinase of JNK. SEK1 was basally phosphorylated in rat hippocampus. In contrast to the increase of the phosphorylation of JNK, SEK1 was gradually dephosphorylated in rat hippocampus after KA treatment. The dephosphorylation of SEK1 was apparent at $1 \mathrm{~h}$ and phospho-SEK1 band was barely seen at $3 \mathrm{~h}$ (Figure $1 \mathrm{~B}$, right). These results suggested the possibility that SEK1 may not be the JNK kinase in rat brain (Oh et al., 1999). Reports of ischemia-induced down-regulation of p38 activity in rat liver and our data suggest that the down-regulation of SEK1 activity following KA treatment in rat hippocampus itself may be a signaling transducer. It remains to be elucidated that whether the SEK1 in rat brain has another substrate other than JNK, and what is the upstream kinase for the activation of JNK in rat hippocampus after KA treatment. KA also increased the phosphorylation of MKK6 in rat hippocampus, which is known as the upstream kinase of p38. The phosphorylation of MKK6 was observed at $30 \mathrm{~min}$, reached peak at $2 \mathrm{~h}$ and lasted until $3 \mathrm{~h}$, when the phosphorylation of p38 was barely detected in hippocampus (Figure 1C, right). In contrast to MKK6, MKK3 was basally phosphorylated in hippocampus and slight dephosphorylation was observed at $2 \mathrm{~h}$ after KA treatment (Figure $1 C$, right). These results indicate the possibility that MKK3 and MKK6 may not be the p38 kinases and play distinct roles in rat brain.

\section{Discussion}

Previously we reported that KA administration activated 42 and 44 kD MAPKs in rat hippocampus (Kim et al., 1994). In this study, we showed that the other members of MAPKs family, JNK and p38, were also activated in rat hippocampus after KA treatment. Our results indicate that $\mathrm{KA}$ activates all of the three members of MAPK family in rat hippocampus. But, the durations of their activation were markedly different. ERK activation lasted until $6 \mathrm{~h}$ after $\mathrm{KA}$ treatment, as long as the seizures continued, and returned to the basal level when the acute seizure disappeared at $12 \mathrm{~h}$ (data not shown). The activation of JNK and p38 were transient; returned to the basal level by $2 \mathrm{~h}$ after KA treatment. Our data support a previous in vitro data which demonstrated the activation of ERKs, p38 and JNK by KA in primary hippocampal neurons (DeCoster et al., 1998). But, recently, Mielke et al. (1999) reported a prolonged JNK activation and a persistent down-regulation of p38 activity in nuclear fraction of rat hippocampus after KA administration, which differed from our results. A possible explanation for the difference may be that they assayed the activities of JNK and p38 in nuclear fractions whereas our assays were done in total lysate. The JNK translocated to the nucleus after activation persistently phosphorylated and do not return to cytosol while the rest of JNK, which are in cytoplasm, are dephosphorylated. Therefore it will be interesting to see whether the phosphorylation of target transcription factors such as cJun in rat hippocampus after KA administration is persistent or transient.

JNK and p38 are known to be involved in cell death induced by deprivation of nerve growth factor in PC12 cells, and by ceramide in U937 (Xia et al., 1995; Karasavvas and Zakeri 1999). In rat cerebellar granular cells, p38 has been reported to be important in glutamate-induced apoptosis (Kawasaki et al., 1997). Our results suggested that the KA-induced activation of JNK and p38 may play an important role in the induction of neuronal cell death. However, KA administration also induced the prolonged activation of ERKs, which is generally considered as anti-apoptotic signal. ERKs have been reported to be enhanced by stimulation of metabotropic and kainate types of glutamate receptors but not by stimulation of the NMDA receptor (Fiore et al., 1993). Therefore, it is not clear why the activation of KA receptor activates both proliferative and apoptotic signal simultaneously, unlike the activation of NMDA receptor which activates only apoptotic signal by JNK and p38.

$\mathrm{KA}$ administration in cultured neuronal cell and in vivo intracerebral injection experiment showed that the direct activation of AMPA/KA receptor may induce the neuronal cell death (Koh et al., 1990; Simonian et al., 1996). But, an involvement of NMDA receptor in the KA-induced cell death has been suggested, since the NMDAreceptor blockade, MK-801, was reported to have a neuroprotective effect against KA-induced neuronal death (Clifford et al., 1990; Ferrer et al., 1995; Fujikawa et al., 2000). This suggests that KA may induce endogeneous glutamate release which is responsible for the activation of JNK and p38 and also seizure-induced neuronal cell death. Since KA administration activated all of the three MAPK family kinases in rat hippocampus, it may be possible that the KA activates MAPK family kinases by direct activation of KA/AMPA receptor and also through release of glutamate and activation of NMDA receptor.

Previously, we suggested that SEK1 and MKK3 may not be the upstream kinases for JNK and p38 in rat hippocampus after electroconvulsive shock. Our results 
are in support of our previous observation. SEK1 and MKK3 were dephosphorylated gradually after KA administration while the phosphorylation of p46 JNK and p38 were increased. The phosphorylation of MKK6, another upstream kinase of $\mathrm{p} 38$, reached peak at $2 \mathrm{~h}$ after $\mathrm{KA}$ adminstration, when the phosphorylation of p38 returned to the basal level, which also suggested that MKK6 may not be the kinase for p38 in rat hippocmapus. Together with our previous results, our present data clearly indicate that MAPK kinases (MEK) other than SEK1, MKK3 or MKK6 are responsible for the activation of JNK and p38 in rat hippocampus after electroconvulsive shock or KA administration. Further studies are needed to clarify the kinases for the activation of JNK and p38 in rat hippocampus by KA receptor activation.

In conclusion, we demonstrated that intraperitoneal administration of KA activates all of the three MAPK family kinases in rat hippocampus with different temporal patterns and unknown MAPK kinases are involved in the activation of JNK and p38.

\section{Acknowledgements}

This study was supported in part by the Grant for Basic Medical Research from Ministry of Education (KRF-97021-F00097).

\section{References}

Casanova, E., Garate, C., Ovalle, S., Calvo, P. and Chinchetru, M. A. (1996) Identification of four splice variants of the mouse stress-activated protein kinase JNK/SAPK alpha-isoform. Neuroreport. 7: 1320-1324

Cheng, H. L. and Feldman, E. L. (1998) Bidirectional regulation of p38 kinase and C-Jun N-terminal protein kinase by insulin-like growth factor-I. J. Biol. Chem. 273: 14560-14565

Choi, D. W. (1988) Glutamate neurotoxicity and diseases of the nervous system. Neuron 1: 623-634

Clifford, D. B., Olney, J. W., Benz, A. M., Fuller, T. A. and Zorumski, C. F. (1990) Ketamine, phencyclidine, and MK-801 protect against kainic acid-induced seizure-related brain damage. Epilepsia 31: 382-390

DeCoster, M. A., Mukherjee, P. K., Davis, R. J. and Bazan, N. G. (1998) Platelet-activating factor is a downstream messenger of kainate-induced activation of mitogen-activated protein kinases in primary hippocampal neurons. J. Neurosci. Res. 53: $297-303$

Ferrer, I., Planas, A. M. and Pozas, E. (1997) Radiationinduced apoptosis in developing rats and kainic acid-induced excitotoxicity in adult rats are associated with distinctive morphological and biochemical c-Jun/AP-1 (N) expression. Neuroscience 80: 449-458
Fiore, R. S., Murphy, T. H., Sanghera, J. S., Pelech, S. L. and Baraban, J. M. (1993) Activation of p42 mitogen-activated protein kinase by glutamate receptor stimulation in rat primary cortical cultures. J. Neurochem. 61: 1626-1633

Fujikawa, D. G., Shinmei, S. S. and Cai, B. (2000) Kainic acidinduced seizures produce necrotic, not apoptotic, neurons with internucleosomal DNA cleavage: implications for programmed cell death mechanisms. Neuroscience 98: 41-53

Gupta, S., Barrett, T., Whitmarsh, A. J., Cavanagh, J., Sluss, H. K., Derijard, B. and Davis, R. J. (1996) Selective interaction of JNK protein kinase isoforms with transcription factors. EMBO J. 15: 2760-2770

Karasavvas, N. and Zakeri, Z. (1999) Relationships of apoptotic signaling mediated by ceramide and TNF-alpha in U937 cells. Cell Death Differ. 6: 115-123

Kawasaki, H., Morooka, T., Shimohama, S., Kimura, J., Hirano, T., Gotoh, Y. and Nishida, E. (1997) Activation and involvement of p38 mitogen-activated protein kinase in glutamate0induced apoptosis in rat cerebellar granule cells. J. Biol. Chem. 272: 18518-18521

Kim, Y. S., Hong, K. S., Seong, Y. S., Park, J. B., Kuroda, S., Kishi, K., Kaibuchi, K. and Takai, Y. (1994) Phosphorylation and activation of mitogen-activated protein kinase by kainic acid-induced seizure in rat hippocampus. Biochem. Biophys. Res. Commun. 202: 1163-1168

Koh, J. Y., Goldberg, M. P., Hartley, D. M. and Choi, D. W. (1990) Non-NMDA receptor-mediated neurotoxicity in cortical culture. J. Neurosci. 10: 693-705

Mielke, K., Brecht, S., Dorst, A. and Herdegen, T. (1999) Activity and expression of JNK1, p38 and ERK kinases, c-Jun $\mathrm{N}$-terminal phosphorylation, and c-jun promoter binding in the adult rat brain following kainate-induced seizures. Neuroscience 91: 471-483

Nicoll, R. A., Kauer, J. A. and Malenka, R. C. (1988) The current excitement in long-term potentiation. Neuron 1: 97-103

Oh, S. W., Ahn, Y. M., Kang, U. G., Kim, Y. S. and Park, J. B. (1999) Differential activation of c-Jun $\mathrm{N}$-terminal protein kinase and p38 in rat hippocampus and cerebellum after electroconvulsive shock. Neurosci. Lett. 271: 101-104

Robinson, M. J. and Cobb, M. H. (1997) Mitogen-activated protein kinase pathways. Curr. Opin. Cell Biol. 9: 180-186

Seger, R. and Krebs, E. G. (1995) The MAPK signaling cascade. FASEB J. 9: 726-735

Simonian, N. A., Getz, R. L., Leveque, J. C., Konradi, C. and Coyle, J. T. (1996) Kainic acid induces apoptosis in neurons. Neuroscience 75: 1047-1055

Widmann, C, Gibson, S, Jarpe, M. B. and Johnson, G. L. (1999) Mitogen-activated protein kinase: conservation of a three-kinase module from yeast to human. Physiol. Rev. 79: 143-180

Xia, Z., Dickens, M., Raaingeaud, J., Davis, R. J. and Rincon, M. E. (1995) Opposing effects of ERK and JNK-p38 MAPkinases on apoptosis. Science 270: 1326-1331 